\title{
The BMP Coreceptor RGMb Promotes While the Endogenous BMP Antagonist Noggin Reduces Neurite Outgrowth and Peripheral Nerve Regeneration by Modulating BMP Signaling
}

\author{
Chi H. E. Ma, ${ }^{1,7}$ Gary J. Brenner, ${ }^{2}$ Takao Omura, ${ }^{1}$ Omar A. Samad, ${ }^{4}$ Michael Costigan, ${ }^{1}$ Perrine Inquimbert, ${ }^{1}$ \\ Vera Niederkofler, ${ }^{5}$ Rishard Salie, ${ }^{5}$ Chia Chi Sun, ${ }^{3}$ Herbert Y. Lin, ${ }^{3}$ Silvia Arber, ${ }^{4}$ Giovanni Coppola, ${ }^{6}$ Clifford J. Woolf, ${ }^{1}$ \\ and Tarek A. Samad ${ }^{8}$ \\ ${ }^{1}$ F.M. Kirby Neurobiology Center, Children's Hospital Boston, Harvard Medical School, Boston, Massachusetts 02115, ${ }^{2}$ Department of Anesthesia, Critical \\ Care and Pain Medicine, and ${ }^{3}$ Center for Systems Biology, Program in Membrane Biology and Division of Nephrology, Department of Medicine, \\ Massachusetts General Hospital and Harvard Medical School, Boston, Massachusetts 02114, ${ }^{4}$ Department of Neurology and Center for Neuroscience and \\ Regeneration Research, Yale University School of Medicine, New Haven, Connecticut 06520, ${ }^{5}$ Biozentrum, Department of Cell Biology, University of Basel, \\ 4056 Basel, Switzerland, and Friedrich Miescher Institute for Biomedical Research, 4058 Basel, Switzerland, ${ }^{6}$ Semel Institute for Neuroscience and Human \\ Behavior, Department's of Psychiatry and Neurology, David Geffen School of Medicine, University of California Los Angeles California 90095, ${ }^{7}$ Department \\ of Biology and Chemistry, City University of Hong Kong, Kowloon, Hong Kong, and ${ }^{8}$ Neuroscience Research Unit, Pfizer, Groton, Connecticut 06340
}

Repulsive guidance molecule b (RGMb) is a bone morphogenetic protein (BMP) coreceptor and sensitizer of BMP signaling, highly expressed in adult dorsal root ganglion (DRG) sensory neurons. We used a murine RGMb knock-out to gain insight into the physiological role of RGMb in the $\mathrm{DRG}$, and address whether RGMb-mediated modulation of BMP signaling influences sensory axon regeneration. No evidence for altered development of the PNS and CNS was detected in $R G M b^{-1-}$ mice. However, both cultured neonatal whole DRG explants and dissociated DRG neurons from $R G M b^{-1-}$ mice exhibited significantly fewer and shorter neurites than those from wild-type littermates, a phenomenon that could be fully rescued by BMP-2. Moreover, Noggin, an endogenous BMP signaling antagonist, inhibited neurite outgrowth in wild-type DRG explants from naive as well as nerve injury-preconditioned mice. Noggin is downregulated in the DRG after nerve injury, and its expression is highly correlated and inversely associated with the known regeneration-associated genes, which are induced in the DRG by peripheral axonal injury. We show that diminished BMP signaling in vivo, achieved either through $R G M b$ deletion or BMP inhibition with Noggin, retarded early axonal regeneration after sciatic nerve crush injury. Our data suggest a positive modulatory contribution of $R G M b$ and BMP signaling to neurite extension in vitro and early axonal regrowth after nerve injury in vivo and a negative effect of Noggin.

\section{Introduction}

Cell specification, differentiation, proliferation, patterning and migration during vertebrate development are profoundly influenced by transforming growth factor $\beta$ (TGF $\beta$ ) superfamily li-

\footnotetext{
Received Sept. 6, 2011; revised 0ct. 21, 2011; accepted 0ct. 28, 2011.

Author contributions: G.J.B., C.J.W. and T.A.S. designed research; C.H.E.M., G.J.B., T.O., 0.A.S., M.C., P.I., V.N., R.S., C.C.S., G.C., and T.A.S. performed research; S.A. and T.A.S. contributed unpublished reagents/analytic tools; C.H.E.M., G.J.B., T.O., H.Y.L., S.A., G.C., C.J.W., and T.A.S. analyzed data; G.J.B. and T.A.S. wrote the paper.

This work was supported by the NIH [Grants NS038253 and RDE017821A (C.J.W.), and NS044139 (G.J.B.)], the Dr. Miriam and Sheldon G. Adelson Medical Research Foundation, the Hong Kong Croucher Foundation Fellowship (C.H.E.M.), the Swiss National Science Foundation, an European Research Council Advanced Grant (S.A., V.N. and R.S.), and the IRP-IFP Switzerland (M.C., G.C.). We thank Yin Xia, Qiufu Ma, Kenneth Bloch, and Robert Griffin for their helpful input.

C.H.E.M., G.J.B., and T.O. contributed equally.

The authors declare no competing financial conflicts.

Correspondence should be addressed to either of the following: Dr. Tarek A. Samad, Neuroscience Research Unit, Pfizer, Groton, С 06340. E-mail:Tarek.samad@pfizer.com; or Dr. Gary J. Brenner, Department of Anesthesia, Critical Care and Pain Medicine, Massachusetts General Hospital and Harvard Medical School, Boston, MA 02114. E-mail: Gjbrenner@partners.org.

DOI:10.1523/JNEUROSCI.4550-11.2011

Copyright $\odot 2011$ the authors $\quad 0270-6474 / 11 / 3118391-10 \$ 15.00 / 0$
}

gands, particularly BMPs (Balemans and Van Hul, 2002; Wu and Hill, 2009). These diverse functions require both tight spatiotemporal control of ligand production and activation of specific intracellular signaling pathways by type I and II serine-threonine kinase receptors. BMPs direct skeletal patterning, chondrogenesis and bone formation in the embryo (Babitt et al., 2006; Rosen, 2006), and in the nervous system both act as instructive signals for neuronal lineage commitment and promote neuronal differentiation (Liu and Niswander, 2005).

BMP signaling is greatly enhanced in those cells that express the glycosyl-phosphatidylinositol-anchored repulsive guidance molecule a (RGMa), RGMb, and RGMc BMP coreceptors, sensitizing these cells to low levels of ligand (Severyn et al., 2009). RGMb (also termed "Dragon") is a 436 aa membrane-bound protein expressed in the adult in a wide range of neural sites including the DRG, spinal cord, optic nerve, retina, and several regions of the brain (Niederkofler et al., 2004; Oldekamp et al., 2004; Samad et al., 2004; Severyn et al., 2009), as well as the reproductive tract (Xia et al., 2005) and kidney (Xia et al., 2010). 
RGMb potentiates BMP signaling via direct binding to BMP-2 and BMP-4, and subsequent association with type I and II BMP receptors (Samad et al., 2004). RGMb is expressed in developing mouse bone and nervous system (Samad et al., 2004, 2005) and in the branchial arches, somites and tail bud of developing mouse and Xenopus (Samad et al., 2005). While the role of RGMc (also known as hemojuvelin) in regulating iron homeostasis is well established (Niederkofler et al., 2005; Babitt et al., 2006), less is known about the roles of RGMa and RGMb in the nervous system. RGMa is suggested to contribute to neuronal differentiation and axonal guidance (Matsunaga et al., 2006; Liu et al., 2009; Severyn et al., 2009; Conrad et al., 2010); however, RGMadeficient mice do not display alterations in neuronal patterning and retinal projection topography (Niederkofler et al., 2004).

Several lines of evidence suggest that BMP-signaling may be involved in neuronal regeneration. Specifically, BMP receptors, ligands, and downstream effectors are expressed in the PNS, and there is an injury-induced regulation of these BMP-signaling pathway components (Ai et al., 1999; Tsujii et al., 2009; Zou et al., 2009). Interestingly, BMP signaling appears to have differential effects on neurite extension and axonal growth in the central (Matsuura et al., 2007, 2008; Liu et al., 2009) and peripheral (Hodge et al., 2007; Moon and Birren, 2008; Tsujii et al., 2009) nervous systems, BMP pathway activation appears to be predominantly inhibitory in the CNS and permissive in the PNS.

Here we show that deletion of the $R G M b$ gene in mice or the use of the endogenous BMP signaling antagonist, Noggin, decreases BMP-mediated signaling in primary sensory neurons and alters their axonal regenerative response to injury both in vitro and in vivo. These findings provide further support for BMPsignaling as a growth promoting pathway in the PNS following injury, and insight into the contribution of positive and negative BMP-signaling modifiers in this process.

\section{Materials and Methods}

Animals. All surgical procedures were performed in accordance with Massachusetts General Hospital and Children's Hospital Animal Care guidelines. RGMb KO and WT littermate mice of either sex at postnatal day 8 (P8)-P9 were used in nerve crush and regeneration studies. In the Noggin experiment on adult mice, C57BL/6 adult male mice were obtained from Charles River Laboratories.

Generation and phenotypes of $R G M b \mathrm{KO}$ mice. $R G M b \mathrm{KO}$ mice (C57BL/6/129) lacking exon II of the RGMb gene were generated and were genotyped as described previously (Xia et al., 2011). Heterozygous animals were bred to obtain homozygous $R G M b \mathrm{KO}$ mice; this was necessary as $R G M b$-null animals die at $\sim 21 \mathrm{~d}$ of age. Histological analysis and magnetic resonance imaging of an $R G M b \mathrm{KO}$ mouse and its WT littermate (LM) failed to reveal any gross differences in brain or ventricular structure (data not shown). The KO mice display less pulmonary alveolarization, as well as delayed eruption and poor root formation of the teeth (data not shown).

In situ hybridization and immunohistochemistry. Tissue was rapidly removed, embedded in Tissue-Tek OCT, and frozen. Sections were cut serially at $12 \mu \mathrm{m}$, and in situ hybridization was performed using digoxigenin-labeled antisense and sense riboprobes according to published methods (Blackshaw and Snyder, 1997). $R G M b$ primers were as follows: $5^{\prime}$-TGCCAACAGCCTACTCAATG-3' (RGMb exon $1-3$ ) and 5'-GACCAACAGGCAAATCCCTA-3' (RGMb exon 1-3). We detected the hybridized riboprobes using Fab fragments from an anti-digoxigenin antibody conjugated to horseradish peroxidase (Roche). Visualization was conducted using BCIP (Roche) and NBT (Roche) in a solution containing $0.1 \mathrm{~m}$ Tris, $\mathrm{pH} 9.5,0.1 \mathrm{M} \mathrm{NaCl}$, and $50 \mathrm{~mm} \mathrm{MgCl}_{2}$. The probes for Ret, CGRP, Trk A, Trk B, Trk C, Ngn1, Math1, and Mash1 have been described previously (Ma et al., 1997; Abdel Samad et al., 2010). Trk A or neurofilament (NF-200) immunostaining was performed on the same section after in situ hybridization according to published methods (Brenner et al., 2004). Sections were blocked with 4\% donkey serum/ $0.1 \%$ Triton in PBS, and incubated with anti-Trk A (rabbit polyclonal, 1:1000, Millipore Bioscience Research Reagents) or anti-NF-200 (mouse monoclonal, 1:2500, Abcam) antibody. Following primary antibody incubation, sections were incubated with Cy3-conjugated secondary antibody (1:300, Jackson Lab).

Primary DRG culture. P1 DRG cultures were prepared (Benn et al., 2002) and cultured in Neurobasal (Full NB) media supplemented with B27 (Invitrogen), $50 \mathrm{ng} / \mathrm{ml} \mathrm{NGF} \mathrm{(Invitrogen),} 2$ ng/ml GDNF (Sigma), $10 \mu \mathrm{M}$ Ara-C, and $200 \mathrm{~mm}$ L-glutamine. Dissociated DRG neurons were plated onto a poly-D-lysine-laminin-coated 8-well chamber slide (Lab-Tek) for $24 \mathrm{~h}$, washed once, and replaced with fresh NB medium with indicated concentrations of BMP-2. After an additional $24 \mathrm{~h}$, neuronal cultures were fixed with $4 \%$ paraformaldehyde and immunostained with both anti- $\beta$-tubulin III (rabbit monoclonal, 1:1000, Sigma) and antipSmad1/5/8 (rabbit polyclonal, 1:1000, Cell Signaling Technology) for pSmad induction studies. pSmad expression was analyzed by using ImageJ (NIH) to assess signal intensity. For neurite outgrowth assay, neurons were cultured with BMP for $17 \mathrm{~h}$, immunostained with anti- $\beta$ tubulin III, and the longest neurites were measured using ImageJ (NIH). Data were obtained from at least three separate experiments.

Western blot. Cultured DRG neurons were treated with $100 \mathrm{ng} / \mathrm{ml}$ BMP-2 for $1 \mathrm{~h}$ and were sonicated in a lysis buffer, and Western blot was performed as described previously (Samad et al., 2004). Briefly, protein samples were separated on a 4-15\% SDS-PAGE gel (Bio-Rad) and transferred to a polyvinylidene difluoride filter (Immobilon-P; Millipore). The blots were blocked with $6 \%$ nonfat milk and incubated with anti$R G M b$ antibody (rabbit polyclonal, 1:6000), produced in our laboratory as previously described (Samad et al., 2004). The blots were incubated with HRP-conjugated anti-rabbit secondary antibody (1:2000; GE Healthcare), developed in ECL solution (PerkinElmer Life Sciences), and exposed to film.

DRG explant culture. DRGs from P9 RGMb KO and WT (see Fig. 3B) or 8-week-old C57BL/6 (see Fig. 5A) mice were cleaned of spinal and peripheral roots and plated onto 8-well chamber slide dish (Lab-Tek) with thin layer of Matrigel (BD Biosciences). DRGs were cultured in Full $\mathrm{NB}$ media and $50 \mathrm{ng} / \mathrm{ml}$ BMP (see Fig. $3 B$ ) or Noggin $2 \mu \mathrm{g} / \mathrm{ml}$ (see Fig. $5 A$ ) as indicated. After $48 \mathrm{~h}, \mathrm{DRGs}$ were fixed with $4 \%$ paraformaldehyde, washed and then blocked with $2 \%$ goat serum $/ 0.3 \%$ Triton, and immunostained with anti- $\beta$-tubulin III antibody (rabbit monoclonal, 1:500, Sigma). Nonoverlapping quadrant images were taken at low magnification $(4 \times)$ for analysis. The total length of neurite attached to the DRG explants was measured using WIS-NeuroMath (Weizmann Institute of Science).

Sciatic nerve crush. Nerve injury and the in vivo measurement of regeneration were performed under isoflurane anesthesia. Crush injury was performed with smooth forceps (5/45, Fine Scientific Tools), which were used to clamp the sciatic nerve for $30 \mathrm{~s}$ at the level of the proximal thigh. The crush site was marked with a 10-0 nylon suture knotted to the epineurium.

Measurement of axonal regeneration. The sciatic nerve pinch test was used to determine the rate nerve regeneration in vivo (Sjöberg and Kanje, 1990; Mills et al., 2005). At 24 or 48 h after sciatic nerve crush injury animals were anesthetized with $2.5 \%$ isoflurane anesthesia and the left sciatic nerve was exposed. Animals were then brought to a lighter plane of anesthesia ( $0.8 \%$ isoflurane) for measurement of regeneration. Starting distally, a series of pinches using fine smooth forceps (5/45) were delivered to the sciatic nerve, moving proximally toward the injury site. We determined the rate of regeneration by measuring the distance from the injury site, marked with an epineural suture, to the most distal point on the nerve that produced a reflex withdrawal response when pinched. The entire procedure was conducted using a dissecting microscope. For experiments comparing $R G M b \mathrm{KO}$ mice to $\mathrm{LM}$ controls, an individual blind to the pinching assessed reflex withdrawal. GAP-43 fluorescent immunostaining was used for histological characterization of regeneration following crush injury. GAP-43 expression is highly induced in regenerating fibers in the sciatic nerve following injury (Seijffers et al., 2007). Two days after crush injury, sciatic nerves were removed and fixed. Longitudinal 
sections $(10 \mu \mathrm{m})$ were immunostained for anti-GAP-43 (rabbit polyclonal, 1:1000, Millipore Bioscience Research Reagents), images were captured, and the maximal regeneration (as indicated by GAP-43 fiber staining distal to the crush site) was determined.

Peripheral nerve injections. Male C57BL/6 mice were anesthetized with $2.5 \%$ isoflurane and the left sciatic nerve was exposed at the mid-thigh level. Noggin or vehicle was injected directly into the sciatic nerve through a finely pulled glass pipette using a Narishige IM300 Microinjector. A total volume of $1 \mu \mathrm{l}$ was used for noggin $(0.5$ or $1 \mu \mathrm{g}$, see Fig. $5 B ; 2 \mu \mathrm{g}$, see Fig. $5 C$ ) and vehicle injections, all of which were performed blind to substance injected. Sixty seconds after injection, sciatic nerve crush was performed as described above.

Bioinformatic analysis. Weighted gene coexpression network analysis was performed using methods detailed by Oldham et al. $(2006,2008)$ with expression profiling data detailed by Lewis et al. (1999) and Ma et al. (2011). Primary expression array data are deposited in Gene Expression Omnibus (NCBI) GSE30691. Briefly, after selecting genes present in at least five samples, the absolute Pearson correlation coefficients between one gene and every other screened gene were computed, weighted and used to determine the topological overlap, a measure of connection strength, or 'neighborhood sharing' in the network. A pair of nodes in a network is said to have high topological overlap if they are both strongly connected to the same group of nodes. In gene networks, genes with high topological overlap have been found to have an increased chance of being part of the same tissue, cell type or biological pathway. Network Neighborhood Analysis provides a set neighborhood for an initial seed or node. Using Noggin (Affymetrix probe $\mathrm{U} 31203$ ) as the chosen seed, the top 64 nearest neighbors (65 genes total) were selected using topological overlap as a measure of connection strength. Visual $\mathrm{C}++$ implementation of the multinodeTOM software can be found at http://www.genetics.ucla.edu/labs/horvath/MTOM/.

Statistics. Analysis was conducted either by Student's $t$ test or by oneway ANOVA, the latter with post hoc analysis via Newman-Keuls test as appropriate.

\section{Results \\ $R G M b$ deletion alters BMP-mediated Smad1/5/8 phosphorylation in DRG neurons}

$R G M b$ expression starts early in development [before embryonic day 12.5 (E12.5)] and is maintained in adult mouse DRG, spinal cord, and brain (Niederkofler et al., 2004; Samad et al., 2004). In the DRG, RGMb is highly expressed in a subset of small (C-fiber) and large diameter (A- $\beta$ and $A-\delta$ ) neurons as suggested by its partial colocalization with the DRG sensory neuron molecular markers TrkA, Peripherin and Neurofilament 200- expressed in 33,62 , and $20 \%$ of RGMb-positive DRG neurons, respectively (Fig. 1A).

To investigate the role of RGMb in the nervous system, we first determined whether $R G M b$ mutation in mice affects neuronal specification in the DRG. We examined the expression of several well characterized sensory neuron subpopulation markers (Ret, CGRP, TrkA, TrkB, and TrkC) and found no detectable postnatal difference in the phenotype of sensory neurons between $R G M b$ mutant and wild-type mice (Fig. $1 B$ ). Since $R G M b$ is also expressed in the spinal cord, we looked and found no difference in expression of the neurogenic transcription factors Mash1, Math1 and Ngn1 (Ma et al., 1997; Abdel Samad et al., 2010) in the developing spinal cord (E12.5) between $R G M b$ mutant and wildtype (WT) mice (data not shown). These data indicate that $R G M b$ deletion does not appear to affect early neuronal specification of DRG sensory neuron subpopulations.

To test whether lack of $R G M b$ expression in DRG alters BMPmediated signaling in sensory neurons, we exposed cultured neonatal DRG neurons from P14 RGMb KO mice and WT LMs to varying concentrations of BMP-2 and assayed for induction of phospho-Smad1/5/8. Smad1/5/8 proteins, which are activated by phosphorylation, are the primary downstream effectors of BMP receptor activation (Liu and Niswander, 2005). We observed a dose-dependent effect on BMP-2-mediated Smad phosphorylation in cultured DRG neurons from $R G M b \mathrm{KO}$ mice compared with WT LMs (Fig. $2 A, B$ ). pSmad induction in $R G M b$ KO DRG neurons was significantly reduced at $5 \mathrm{ng} / \mathrm{ml}$ (6-fold) and 50 $\mathrm{ng} / \mathrm{ml}$ (3-fold) of BMP-2, but was not different at higher concentrations of BMP-2 (200 ng/ml), when compared with BMP-2 action on neurons from $R G M b$ WT littermates. Consistent with these immunohistochemical data, a marked induction of pSmad by BMP2 was also observed by Western blot in cultured WT DRG neurons (Fig. 2C). Our data indicate that $R G M b$ expression in DRG neurons enhances signaling of BMP ligands, and support a role for RGMb as a BMP sensitizer in sensory neurons, similar to the role of hemojuvelin (RGMc) - another member of the RGM family - in hepatocytes (Babitt et al., 2006).

\section{$R G M b$ deletion and inhibition of BMP signaling by Noggin reduce neurite formation in DRG neurons in vitro}

Smad1, a key intracellular effector required for BMP-2 and -4 signaling, is required for the initiation of sensory neuron axonal regeneration following injury in adult mice (Zou et al., 2009). To test whether RGMb, which can potentiate BMP signaling and hence Smad activation, enhances axonal growth in DRG neurons, we prepared both dissociated DRG neuronal cultures and DRG explants from RGMb mutant mice and their WT LMs. The dissociated neuron cultures allow assessment of neurite formation in individual cells, while the cultured whole explanted DRG maintain important interactions between resident neurons and supporting cells. Dissociated DRG neurons from $R G M b \mathrm{KO}$ mice developed shorter neurites, compared with LM controls $(p<$ 0.001 ) (Fig. 3A). The addition of BMP-2 to cultured RGMbdeficient DRG neurons fully restored average neurite length; there was no significant difference of average length between $R G M b \mathrm{KO}+\mathrm{BMP}-2$ and WT LM (Fig. 3A).Analogous results were obtained using cultured whole DRG explants. $R G M b \mathrm{KO}$ mice exhibited $50 \%$ less neurite outgrowth than explants from WT LMs, and more importantly, this loss of function could be fully rescued by addition of exogenous BMP-2 (Fig. 3B, KO vs $\mathrm{WT} p<0.001 ; \mathrm{KO}$ vs KO-BMP $p=0.001)$. There was no significant effect of the addition of BMP-2 to dissociated WT DRG neurons or to WT DRG explants (Fig. 3). The apparent lack of effect of BMP-2 on cultured WT DRG neurons suggests that there may be a low ceiling tonic BMP signaling effect on neurite outgrowth in vitro such that additional BMP ligand has no further effect. To further test whether the reduced regenerative capacity in DRG explants from $R G M b$-deficient mice is due to decreased BMP signaling, we cultured wild-type naive and preconditioned (i.e., $48 \mathrm{~h}$ after sciatic nerve crush) adult mouse DRG explants with and without the endogenous BMP-inhibitor, Noggin (see Fig. 5A). Exposure to Noggin caused a $60 \%$ decrease in neurite formation in both naive and preconditioned DRG explants $(p<$ $0.01, n=8$ per group) (see Fig. $5 A, B$ ). Overall, these data show that $R G M b$ expression enhances BMP-dependent neurite outgrowth in DRG neurons in vitro and that Noggin reduces neurite outgrowth.

\section{$R G M b$ deficiency and inhibition of BMP signaling by Noggin lead to slower axonal regeneration following sciatic nerve injury}

We next assessed whether $R G M b$ deficiency alters axonal regeneration in vivo after sciatic nerve injury. We used the sciatic nerve crush model for assessment of axonal regeneration in sensory 
A

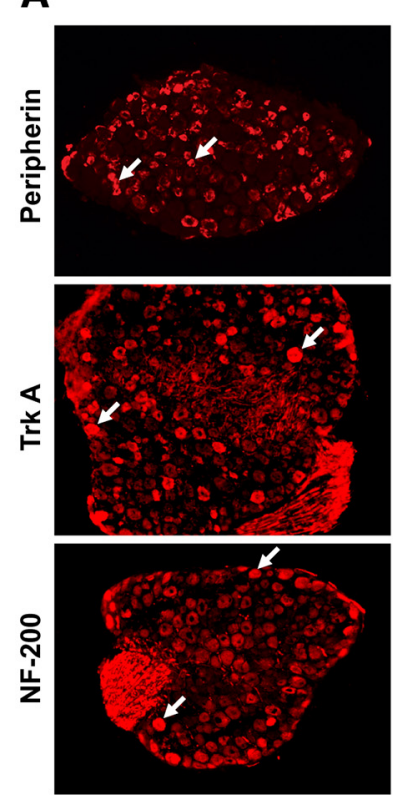

RGMb in situ

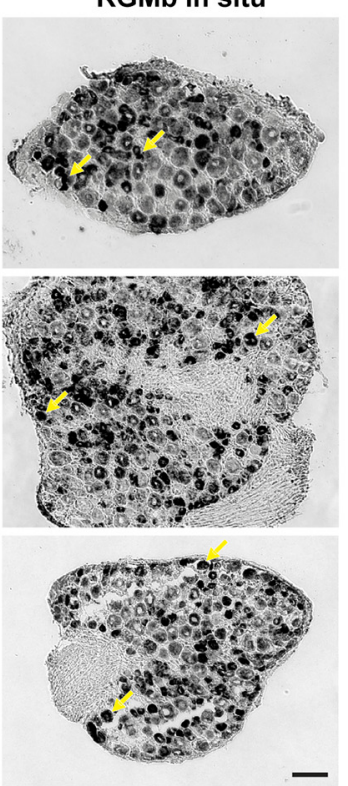

B
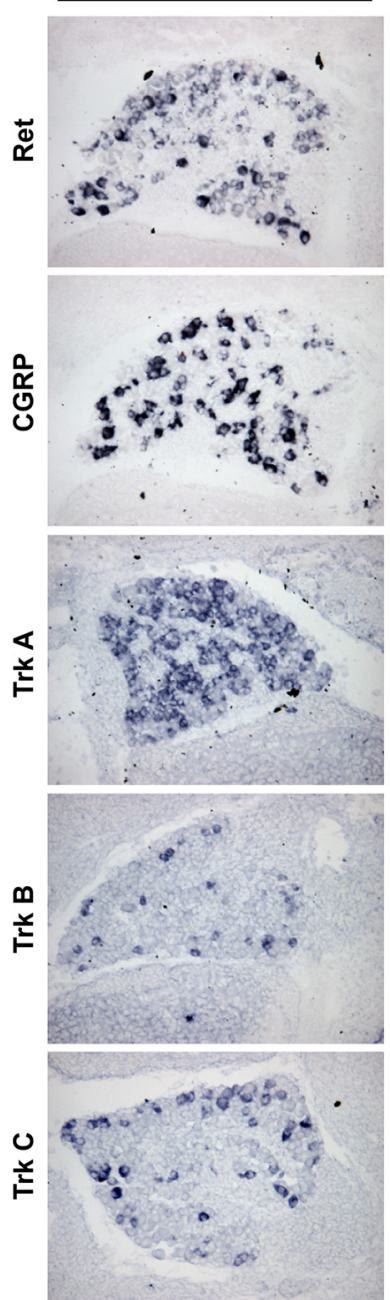

RGMb -/-
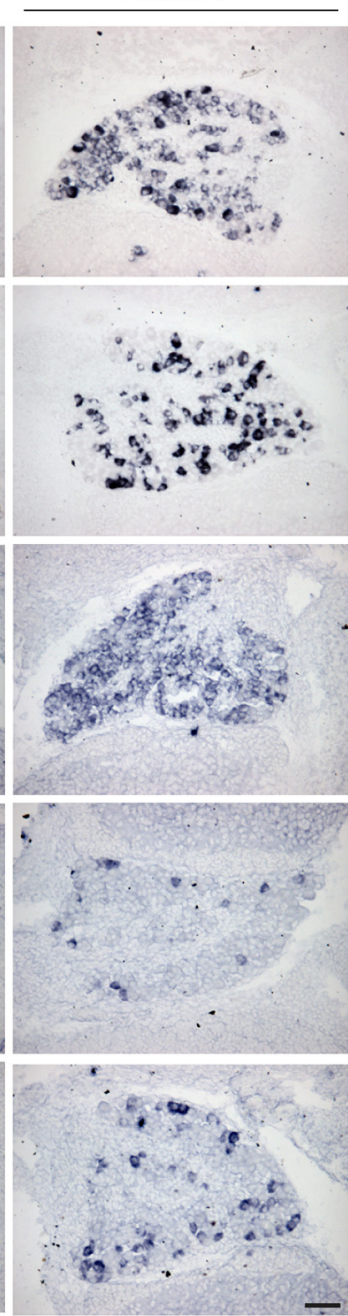

Figure 1. RGMb deletion does not alter development of DRG neurons as revealed by in situ hybridization. $A, R G M b$ is widely expressed in small and large DRG neurons of adult C57BL/6 mice as demonstrated by coexpression with the sensory neuron markers peripherin, TrkA and NF-200, visualized by fluorescent immunohistochemistry (right) and in situ hybridization (left). Arrows indicate cells that express peripherin, TrkA, or NF-200 (white arrows) and RGMb (yellow arrows). Scale bar, $100 \mu \mathrm{m}$. B, In situ hybridization revealed no difference between RGMb K0 and WT LM in the expression of a variety of key markers of sensory neurons in PO DRG.

neurons (Sjöberg and Kanje, 1990). Axonal regrowth in the sciatic nerve following crush injury was assayed both via immunohistochemical evaluation of GAP-43-expressing fibers (Seijffers et al., 2006) and by a functional measure, the pinch test (Seijffers et al., 2007). As a control, we assessed axonal density in the sciatic nerves of $R G M b \mathrm{WT}$ and $\mathrm{KO}$ mice and found no significant difference (KO/WT ratio of neurofilament 200 positive fibers: $0.93 \pm 0.072, p=0.568, n=9$ sections per group, 3 animals per genotype), thus suggesting that there are no major defects in axonal development in the PNS. GAP-43 expression revealed an average of $1.3 \pm 0.1 \mathrm{~mm}$ regrowth in $R G M b \mathrm{KO}$ mice compared with $2.5 \pm 0.2 \mathrm{~mm}$ in WT LM controls $48 \mathrm{~h}$ following crush injury (Fig. $4 \mathrm{~A}$ shows representative images; $p<0.01, n=3$ per group). The pinch test similarly demonstrated delayed axonal regeneration in $R G M b \mathrm{KO}$ animals compared with their WT LMs; Two-way ANOVA of these data indicated a significant one-way effect of $R G M b$ genotype (KO vs WT, $F_{(1,13)}=35.8, p<0.001$ ) and of time after crush ( 24 vs $\left.48 \mathrm{~h}, F_{(1,13)}=47.9, p<0.001\right)$, but no significant interaction between the two (Fig. $4 B, n=8$ and 9 for $\mathrm{KO}$ and WT, respectively). Of note, there was no significant difference in regeneration between $R G M b$ heterozygous (mean $3.3 \pm 0.3 \mathrm{~mm}, n=5)$ and WT $(2.7 \pm 0.1 \mathrm{~mm}, n=5)$ mice at $48 \mathrm{~h}(p=0.25)$.

The diminished axonal regrowth in $R G M b \mathrm{KO}$ mice was not due to DRG cell death as there is no detectable apoptosis in DRG from $R G M b \mathrm{KO}$ mice 2 and $7 \mathrm{~d}$ after sciatic nerve crush in vivo as assessed by TUNEL staining (data not shown). Our in vitro data suggest that the reduced regenerative capacity of DRG neurons and explants from $R G M b$-deficient mice is due to decreased BMP signaling. To test this hypothesis in vivo, we injected the sciatic nerve of WT adult mice with 0.5 or $1 \mu \mathrm{g}$ of Noggin immediately before a sciatic nerve crush. Assessment of regeneration using the pinch test $48 \mathrm{~h}$ after crush injury revealed a significant inhibition ( $p<0.05, n=6)$ associated with Noggin treatment (at both doses) compared with vehicle-injected control mice (Fig. $5 B$ ). A similar effect of Noggin injection $(2 \mu \mathrm{g})$ on in vivo axonal regeneration was found at $4 \mathrm{~d}$ after crush (Vehicle: $6.55 \pm 0.3 \mathrm{~mm}$; Noggin: $3.08 \pm 0.72 \mathrm{~mm} ; p<0.01, n=6$ ). These data suggest that BMP signaling is required for axonal regrowth following sciatic nerve crush, and that RGMb as a BMP signaling enhancer, plays a pivotal role in the BMP effect on early axonal regeneration. 
A

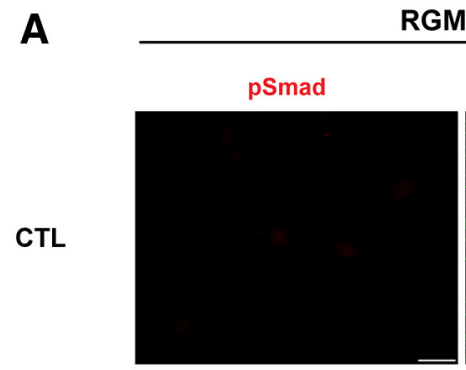

$\mathrm{RGMb}+/+$
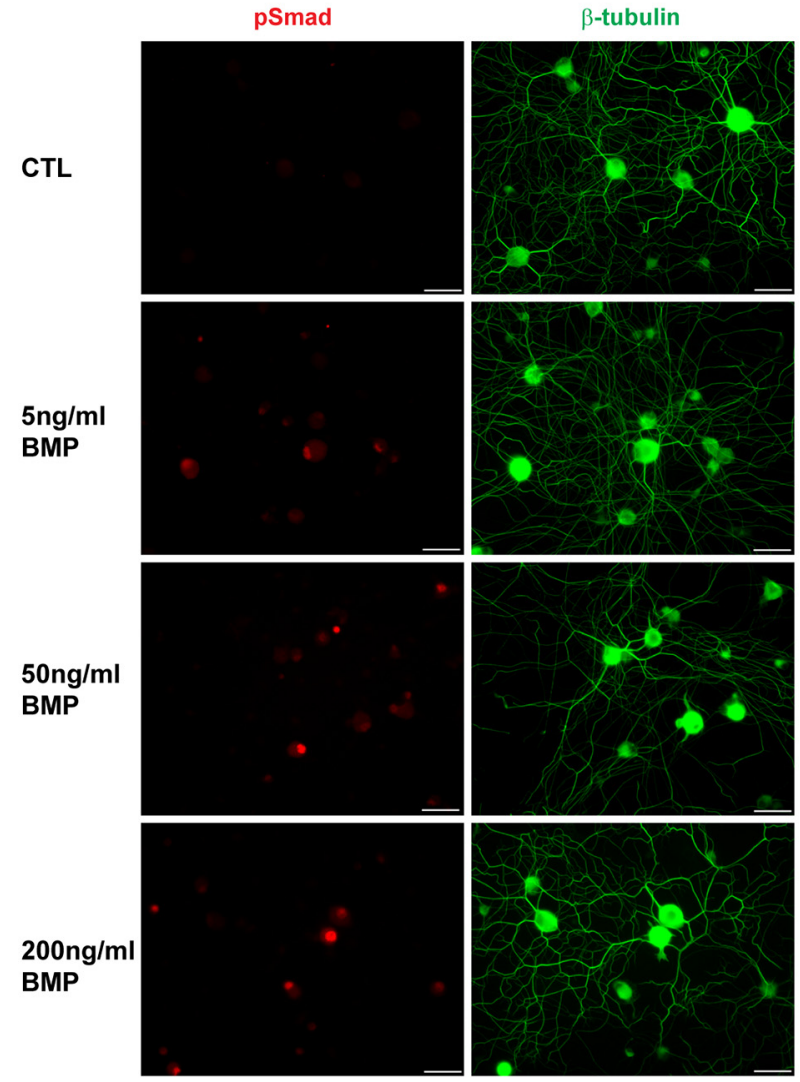

B

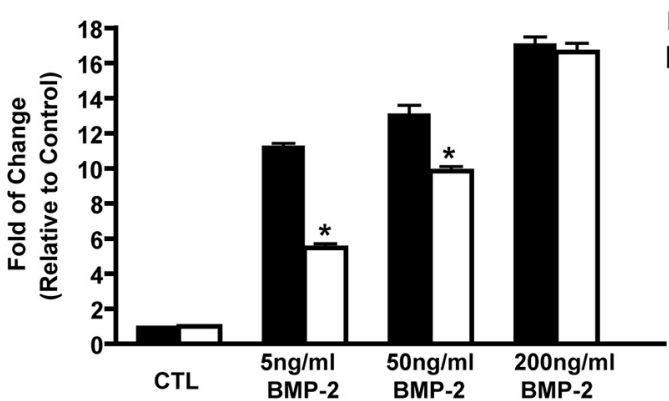

RGMb $=$
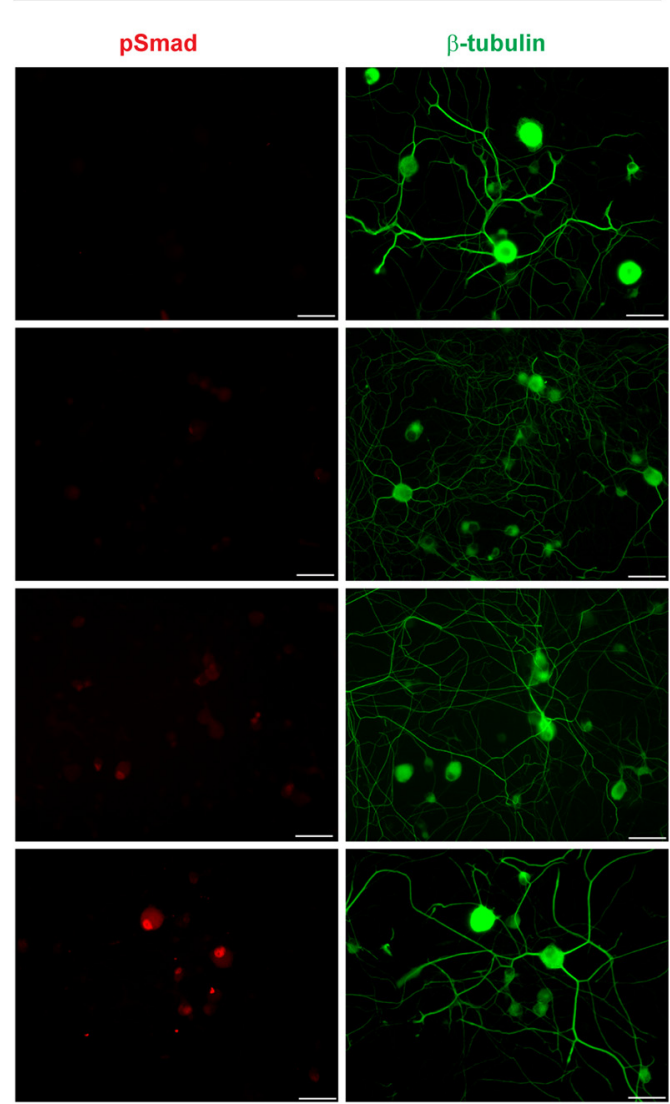

$\mathbb{R}^{+/+} \quad \mathbf{C}$

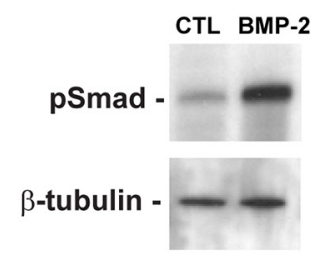

Figure 2. Cultured DRG neurons from RGMb-deficient mice have decreased BMP-2-mediated signaling. DRG neurons from RGMb-deficient and WT LMs were cultured in varying concentrations of BMP-2 and then assayed for phospho-Smad (pSMAD) expression. $\boldsymbol{A}$, Dissociated DRG neurons were immunostained for anti-pSmad (red) and anti- $\beta$-tubulin (green). Scale bars, $10 \mu \mathrm{m}$. $\boldsymbol{B}$, Quantification of $p S$ mad intensity. Values represent mean \pm SEM. ${ }^{*} p<0.001$, statistically significant difference from WT LM. C, Western blot demonstrating BMP-2 (100 ng $/ \mathrm{ml}$ for $\left.1 \mathrm{~h}\right)$ increased pSmad1/5/8 induction in cultured DRG neurons from adult WT mice. $\beta$-Tubulin was used as a loading control.

Noggin expression after peripheral nerve injury negatively correlates over time with the expression of a cluster of regeneration-associated genes

We explored bioinformatically, using a network neighborhood analysis (NNA) (Oldham et al., 2006, 2008), how the pattern of Noggin expression after peripheral nerve injury in the DRG correlates over time with the expression of $\sim 1200$ genes whose expression alters significantly due to nerve injury (Lewis et al., 1999). Of the 65 most closely related genes identified by this analysis (Fig. 6), including Noggin as seed, only one other gene was downregulated (Lin7b) and 97\% were upregulated (63 genes). Remarkably, 29 of 30 genes whose expression across multiple datasets most closely resembled the expression pattern of Hspb1, a newly defined regeneration-associated gene (RAG) (Ma et al., 2011) were present in this list. Of the Hspb1-related genes present 18 have been shown in the literature to increase neurite formation (Ma et al., 2011). These RAGs are Arg1, Atf3, C1qb, Camk2d, Crabp2, Fabp5, Gal, Gap43, Gfra1, Gpc1, Hspb1, Jun, Ptpn5, Sox11, Sprrla, Sprr1al, Stmn4, and Tgm1. The inverse correlation between the expression of Noggin, a negative modulator of BMP signaling, and that of a group of RAGs further supports our findings with respect to the contribution of BMP signaling to axonal regeneration after peripheral nerve injury.

\section{Discussion}

$R G M b$ was initially identified in embryonic DRG, and was the first member of the RGM family shown to potentiate BMP signaling by acting as a BMP coreceptor (Samad et al., 2005). $R G M b$ binds directly BMP ligands, associates with BMP type I (ALK2, ALK3, and ALK6) and type II (ActRII and ActRIIB) receptors, and its signaling is reduced by dominant-negative Smad1 and ALK3 or -6 receptors (Samad et al., 2005). The physiological role 
of $R G M b$ in vivo and its functional implications as a BMP sensitizer in DRG neurons have not, however, been elucidated, until now.

Several lines of evidence suggest that BMP signaling plays a pivotal role in neuronal development; genetic mouse models with null mutations of genes encoding BMPs, BMP receptors, and their downstream signal transducers indicate such a role at several developmental stages, especially for neural patterning (Zhao, 2003). $R G M b$ is widely expressed in the nervous system, mostly in regions complementary to and nonoverlapping with $R G M a$ (Niederkofler et al., 2004; Oldekamp et al., 2004; Samad et al., 2004). Both, RGMa and $R G M b$, act as BMP coreceptors, and their expression levels are upregulated after nerve injury (Schwab et al., 2005). These common patterns suggest potential involvement in comparable physiological roles. Interestingly, while RGMa inhibition has been shown to promote axonal growth and recovery after spinal cord injury (Hata et al., 2006), RGMa expression was shown to promote retinal ganglion cell survival (Koeberle et al., 2010). In addition to RGMs, BMP-2, -4 and -7 as well as their downstream effector pSmad are upregulated after spinal cord injury (Xiao et al., 2010). Increased levels of pSmad1/ $5 / 8$ in neurons, astrocytes and oligodendrocytes following injury, indicates that BMP signaling pathways are likely involved in the cellular response to CNS injury. The selective neural expression pattern of $R G M b$ and its high levels in developing and adult DRG neurons led us to assess the role of this BMP coreceptor in the development and growth of sensory neurons.

We used an $R G M b$-deficient mouse line to study the role of $R G M b$ in DRG neurons and its contribution to axonal growth following injury. We found no evidence for a major developmental defect in sensory neurons in $R G M b \mathrm{KO}$ mice, and our data show that deletion of $R G M b$ leads to impaired DRG neurite outgrowth in vitro and axonal regeneration in vivo. These data are in keeping with previous studies showing BMP involvement in these phenomena (Tsujii et al., 2009; Zou et al., 2009). Injury-induced axonal growth was shown to require the function of the BMP signaling effector Smad1. After peripheral axotomy, neuronal Smad1 is upregulated, and phosphorylated Smad1 accumulates in the nucleus. Both events precede the onset of axonal extension (Zou et al., 2009). We show here that $R G M b$, as a BMP coreceptor, contributes to PNS regeneration after nerve

A
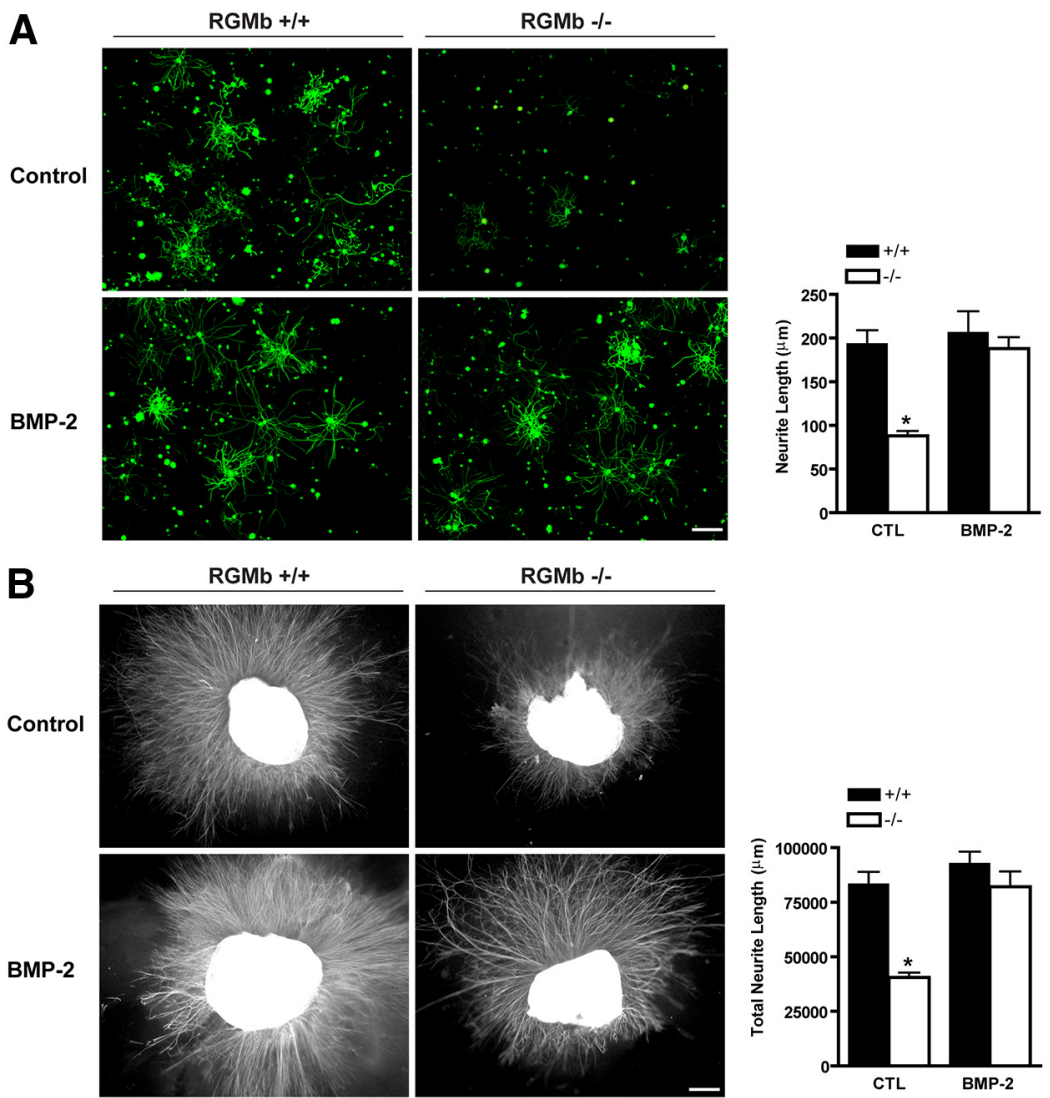

Figure 3. Neurite outgrowth in axotomized $R G M b$ KO DRG neurons is reduced and can be rescued by BMP-2. $A$, Fluorescent microscopy (anti- $\beta$-tubulin immunostaining) of dissociated DRG neurons from RGMb KO and WT LM mice. Fewer cultured DRG neurons from $R G M b^{-1-}$ mice form neurites, and neurites that do form are shorter than those from neurons derived from WT LMs $\left({ }^{*} p<0.001\right)$. Addition of BMP-2 $(50 \mathrm{ng} / \mathrm{ml})$ to the $R G M b^{-1-}$ neurons reverses this effect (no significant difference compared with $R G M b^{+/+}$). Scale bar, $250 \mu \mathrm{m}$. B . Fluorescent micrographs demonstrating that cultured whole DRG explants from $R G M b^{-1-}$ mice generate significantly fewer neurites than explants from WT LM mice and that this loss of function is reversed by the addition of BMP-2 $(50 \mathrm{ng} / \mathrm{ml}) .{ }^{*} p<0.001$, statistically significant difference from all other groups. Values represent mean \pm SEM as shown in all graphs. Scale bar, $250 \mu \mathrm{m}$.

A
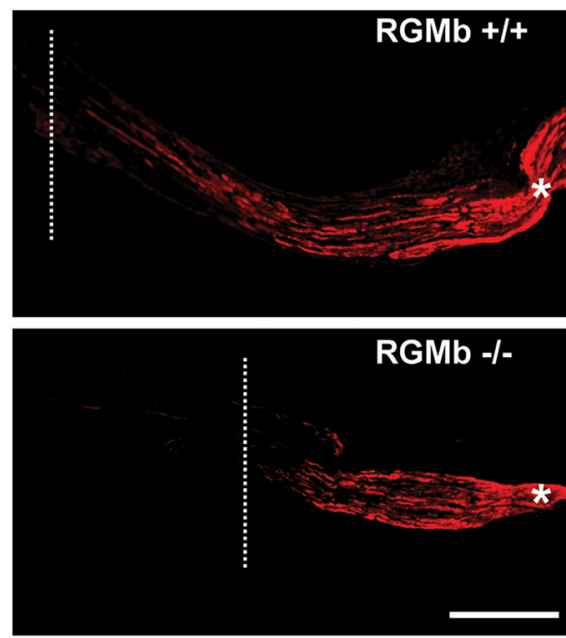

Figure 4. RGMb-deficient mice show retarded axonal regeneration following sciatic nerve crush. $\boldsymbol{A}, \mathrm{GAP}-43$ fluorescent immunohistochemistry demonstrates decreased axonal growth $48 \mathrm{~h}$ following sciatic nerve crush in $R G M b$ KO mice compared with WT LMs; sciatic nerve crush injury was performed at postnatal day 8. Scale bar, $500 \mu \mathrm{m}$. B, Functional regeneration assessed using the pinch test shows decreased regeneration at 24 and $48 \mathrm{~h}$ postinjury in $R G M b$ KO mice compared with WT LMs; sciatic nerve crush injury was performed at postnatal day $8 .{ }^{*} p<0.001$. 

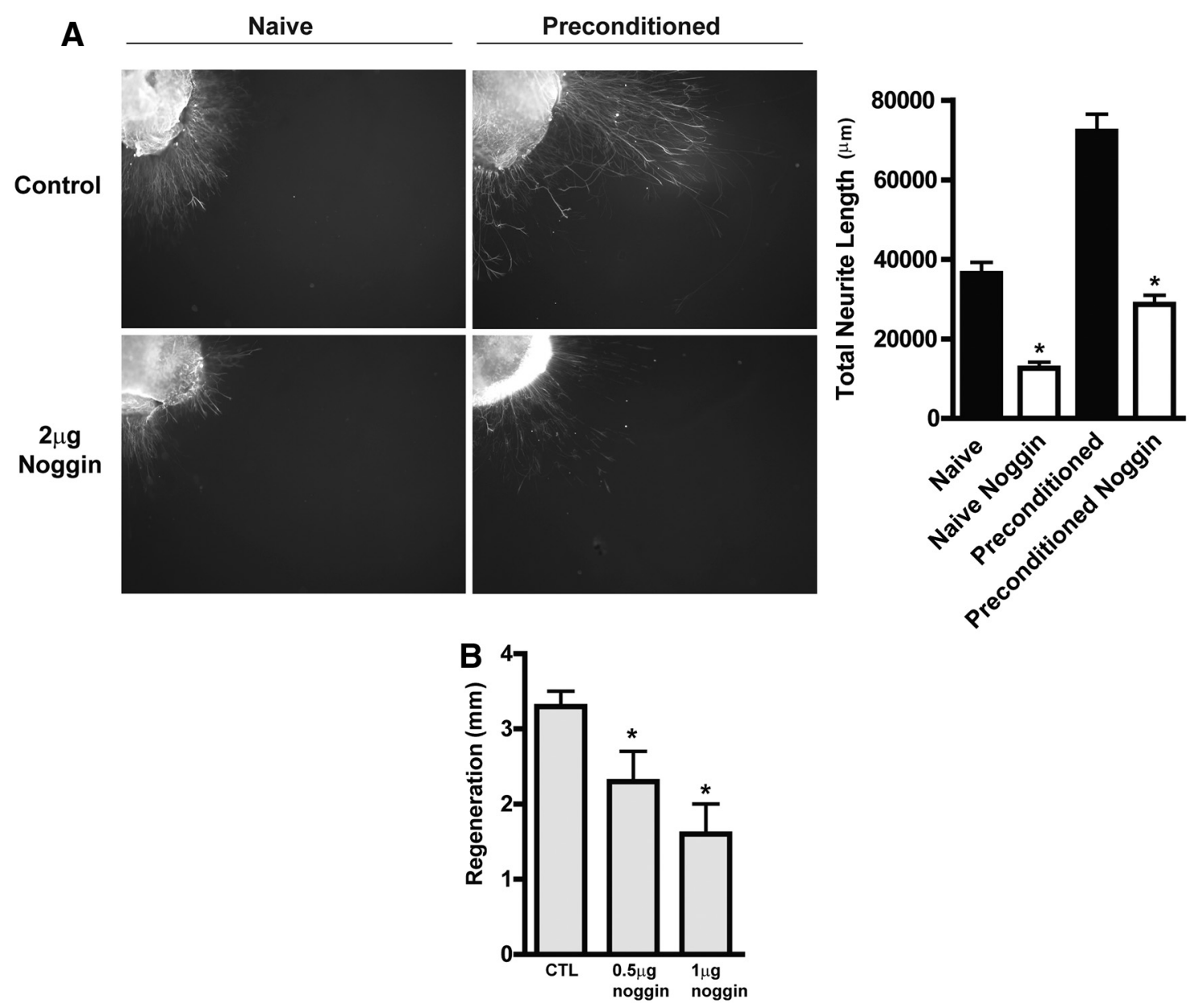

Figure 5. Inhibition of BMP signaling using the endogenous inhibitor, noggin, reduces neurite formation in vitro and axonal regeneration in vivo. $A$, Addition of noggin to culture media causes decreased neurite formation in both naive and preconditioned DRG explants from adult mice ( ${ }^{*} p<0.001, n=8$ per group). $\boldsymbol{B}$, Intra-sciatic injection of the BMP inhibitor, noggin, at both 0.5 and $1 \mu \mathrm{g}$ decreased axonal regeneration $48 \mathrm{~h}$ postinjury as assayed by the pinch test. ${ }^{*} p<0.05$, statistically significant difference from control (CTL), one-way ANOVA followed by post hoc Newman-Keuls test. $n=6$ per group. Values represent mean \pm SEM as shown in all graphs.

injury via the BMP signaling pathway. Since the nerve injury experiments were conducted at P8 in the mutant mice, an age at which the PNS is still developing (Court et al., 2004), we looked at the effect of Noggin in the adult to address a possible contribution of a developmental disorder in the postnatal RGMb mutant mice. We show that Noggin functions as a negative regulator of axonal regeneration in the PNS; it inhibits neurite outgrowth in embryonic and adult DRG neurons and explants in vitro, and reduces adult peripheral nerve regrowth after crush in vivo.

Although in vivo intra-DRG injection of BMP2 was shown to promote axonal growth (Zou et al., 2009), we found that increasing BMP signaling above basal levels did not promote increased neurite outgrowth in WT DRG neurons in vitro, suggesting that the endogenous levels of BMP provided under our experimental conditions in vitro are sufficient for enabling normal axonal growth and no further growth is produced beyond this ceiling effect.

Lack of $R G M b$ in $\mathrm{KO}$ mice diminished basal and BMPinduced levels of Smad1/5/8 phosphorylation in DRG neurons, reaffirming the role of this coreceptor as a BMP ligand sensitizer. Our data suggest that the reduction of BMP signaling resulting from lack of $R G M b$ expression reduced neurite outgrowth in vitro in naive and in injury-preconditioned DRG neurons and explants, by decreasing the responsiveness of the neurons to endogenous BMP. In vivo, $R G M b$ mutation led to reduced axonal regrowth after a sciatic nerve crush injury. That this is likely due to a deficit in BMP signaling is supported by our finding that exogenous administration of the BMP antagonist, Noggin (Smith, 1999) also impaired axonal regeneration in vitro and in vivo. Further, Smad1 phosphorylation and subsequent activation has been implicated in mediating BMP signaling from axon terminals (Hodge et al., 2007), and Smad1 knockdown in adult DRG neurons reduces axonal growth (Zou et al., 2009).

Interestingly, Noggin expression is downregulated in the DRG after nerve injury, as opposed to the upregulation observed for many RAGs. This paradox, due to Noggin's pattern of regulation following nerve injury, closely resembling an inverse plot of the upregulated transcripts that it is grouped with in the NNA (Oldham et al., 2006, 2008), corresponds well with our finding that Noggin as a BMP antagonist negatively regulates neurite formation while induction of the other RAGs increase regeneration. It is entirely possible that other genes whose expression correlates with Noggin may also be therefore RAG genes. The action of $R G M b$ as a coreceptor sensitizing BMP-2 signaling through type I and II BMP receptors is analogous to the role of GFRal (a coreceptor for RET) which sensitizes GDNF signaling and therefore axonal growth within the injured PNS (Mills et al., 2007), interestingly GFRa1 is one of the RAGs inversely associated with Noggin expression. Collectively, these data point to a possible coregulation of the many positive and few negative regulators of axonal 

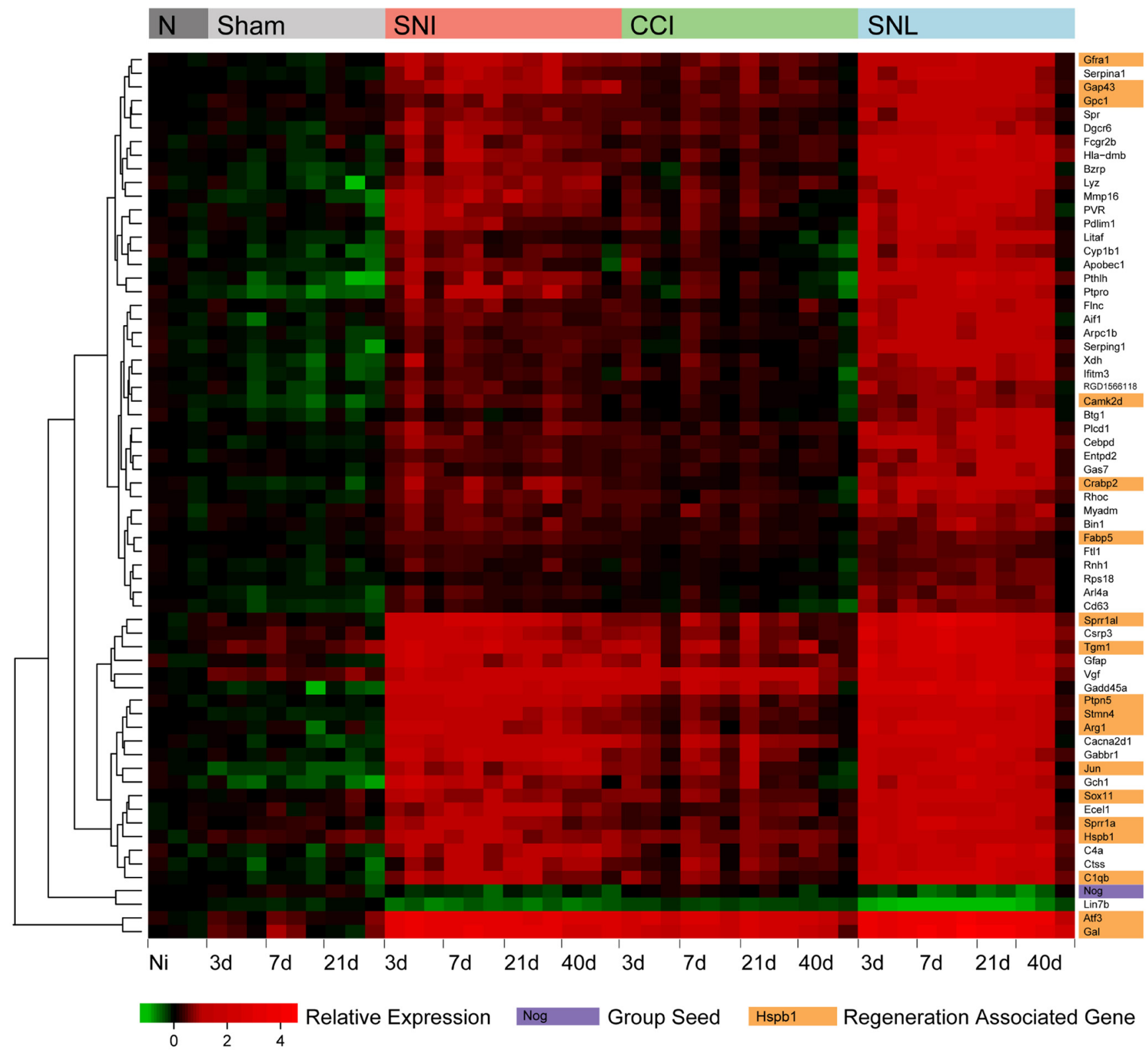

Figure 6. Noggin mRNA expression after peripheral nerve injury negatively correlates over time with the expression of a cluster of regeneration associated genes. Weighted gene coexpression network analysis/neighborhood network analysis was used to determine a group of 65 genes whose expression across an extensive DRG nerve injury dataset most closely relate to Noggin regulation within that group. Heat map showing regulation of these genes in the DRG before (Naive, N) and after sciatic nerve injury [spared nerve injury (SNI), chronic constriction injury (CCI), and spinal nerve ligation (SNL); top colored bar] as well as after sham injury (Sham) over time (3, 7, 21, and $40 \mathrm{~d}$ ) given below. Each colored block within the heat map represents relative expression of that gene in one array in relation to average naive expression. Red on this plot represents upregulated, with green representing downregulated relative to average naive levels. Noggin marked in purple is one of only two downregulated genes present, despite acting as the seed gene whose relative expression across this axonal injury time course was used to choose the most closely related group of 65 genes. The other genes are upregulated after nerve injury but have highly correlated inverse expression patterns to Noggin. To the right are gene names highlighted for function, orange indicates transcripts with a published role in axonal regeneration which have been linked to Hspb1 within (Ma et al., 2011).

growth by common master transcription factors, and that Noggin is the first documented example of the latter and suppression of its expression after injury might contribute to regenerative capacity.

The specific molecular mechanisms by which BMP receptor activation and downstream Smad signaling regulate axonal growth remain poorly understood, Furthermore, a number of BMP-mediated Smad-independent mechanisms have recently emerged as a potential modulators of axonal growth and elongation (Hocking et al., 2009). The actin cytoskeleton in extending axonal and dendritic processes undergoes continuous assembly and disassembly. Extrinsic factors modulate actin turnover through controlling the activity of LIM kinase 1 (LIMK1), which phosphorylates and inactivates the actin depolymerizing factor cofilin. BMPRII is a modulator of LIMK1, and via such an interaction BMPs were shown to induce positive and negative responses in growth cones. BMP ligand binding to the receptor complex modulates this inhibitory interaction and activates Rho GTPase, Cdc42 and LIMK1 (Sánchez-Camacho and Bovolenta, 2009). Further supporting a Smad-independent pathway, expression of a BMPRII mutant lacking the LIMK1 binding region was shown to alter axonal growth (Hocking et al., 2009). Whether the effect of BMP signaling on axonal growth that we observe in $R G M b$-deficient mice is mediated by Smad1 signaling or LIMK1 activation will require additional analysis. 
Our data suggest that RGMb plays a pivotal role in contributing to a tonic level of BMP signaling in DRG neuron that is necessary for normal axonal regrowth. While the neurotrophic properties for BMPs are well established, the tissue distribution and cellular source of these ligands in the peripheral nervous system in vivo have not yet been fully established. In addition to neurons, BMPs (e.g., BMP-2 and -7) and their receptors are expressed in Schwann cells in the sciatic nerve (Tsujii et al., 2009). Schwann cells represent a major source of trophic factors supporting intact and injured neurons. Their responsiveness to BMP ligands represents, therefore, a pathway by which BMPs may modulate neuronal survival and growth indirectly. Interestingly, Schwann cells also express RGMb (data not shown); their contribution to the axonal growth phenotype in $R G M b$ mutant mice cannot be excluded. However, because in dissociated DRG primary cultures lack of neuronal $R G M b$ results in reduced neurite outgrowth, we conclude that neuronal RGMb signaling directly contributes to axonal growth.

Although our data strongly suggest a role for RGMb in promoting BMP signaling and mediating axonal growth, these data do not preclude additional roles for RGMb via BMP-independent mechanisms. It is important to note that RGMa-induced growth cone collapse in DRG neurons is BMP-independent and acts via the GTP-RhoA signaling pathway (Conrad et al., 2007). Noggin also has no effect on RGMa-induced collapse response, further supporting the BMP signaling-independent nature of this phenomena (Conrad et al., 2007).

Together, the data presented here support a role for RGMb, as well as for BMP, signaling in enhancing peripheral nerve regeneration after axonal injury, and suggests that Noggin expression in naive animals may suppress intrinsic growth, an effect relieved by Noggin downregulation after nerve injury.

\section{References}

Abdel Samad O, Liu Y, Yang FC, Kramer I, Arber S, Ma Q (2010) Characterization of two Runx1-dependent nociceptor differentiation programs necessary for inflammatory versus neuropathic pain. Mol Pain 6:45.

Ai X, Cappuzzello J, Hall AK (1999) Activin and bone morphogenetic proteins induce calcitonin gene-related peptide in embryonic sensory neurons in vitro. Mol Cell Neurosci 14:506-518.

Babitt JL, Huang FW, Wrighting DM, Xia Y, Sidis Y, Samad TA, Campagna JA, Chung RT, Schneyer AL, Woolf CJ, Andrews NC, Lin HY (2006) Bone morphogenetic protein signaling by hemojuvelin regulates hepcidin expression. Nat Genet 38:531-539.

Balemans W, Van Hul W (2002) Extracellular regulation of BMP signaling in vertebrates: a cocktail of modulators. Dev Biol 250:231-250.

Benn SC, Perrelet D, Kato AC, Scholz J, Decosterd I, Mannion RJ, Bakowska JC, Woolf CJ (2002) Hsp27 upregulation and phosphorylation is required for injured sensory and motor neuron survival. Neuron 36:45-56.

Blackshaw S, Snyder SH (1997) Parapinopsin, a novel catfish opsin localized to the parapineal organ, defines a new gene family. J Neurosci 17:8083-8092.

Brenner GJ, Ji RR, Shaffer S, Woolf CJ (2004) Peripheral noxious stimulation induces phosphorylation of the NMDA receptor NR1 subunit at the PKC-dependent site, serine-896, in spinal cord dorsal horn neurons. Eur J Neurosci 20:375-384.

Conrad S, Genth H, Hofmann F, Just I, Skutella T (2007) Neogenin-RGMa signaling at the growth cone is bone morphogenetic protein-independent and involves RhoA, ROCK, and PKC. J Biol Chem 282:16423-16433.

Conrad S, Stimpfle F, Montazeri S, Oldekamp J, Seid K, Alvarez-Bolado G, Skutella T (2010) RGMb controls aggregation and migration of Neogeninpositive cells in vitro and in vivo. Mol Cell Neurosci 43:222-231.

Court FA, Sherman DL, Pratt T, Garry EM, Ribchester RR, Cottrell DF, Fleetwood-Walker SM, Brophy PJ (2004) Restricted growth of Schwann cells lacking Cajal bands slows conduction in myelinated nerves. Nature 431:191-195.

Hata K, Fujitani M, Yasuda Y, Doya H, Saito T, Yamagishi S, Mueller BK, Yamashita T (2006) RGMa inhibition promotes axonal growth and recovery after spinal cord injury. J Cell Biol 173:47-58.
Hocking JC, Hehr CL, Bertolesi G, Funakoshi H, Nakamura T, McFarlane S (2009) LIMK1 acts downstream of BMP signaling in developing retinal ganglion cell axons but not dendrites. Dev Biol 330:273-285.

Hodge LK, Klassen MP, Han BX, Yiu G, Hurrell J, Howell A, Rousseau G, Lemaigre F, Tessier-Lavigne M, Wang F (2007) Retrograde BMP signaling regulates trigeminal sensory neuron identities and the formation of precise face maps. Neuron 55:572-586.

Koeberle PD, Tura A, Tassew NG, Schlichter LC, Monnier PP (2010) The repulsive guidance molecule, RGMa, promotes retinal ganglion cell survival in vitro and in vivo. Neuroscience 169:495-504.

Lewis SE, Mannion RJ, White FA, Coggeshall RE, Beggs S, Costigan M, Martin JL, Dillmann WH, Woolf CJ (1999) A role for HSP27 in sensory neuron survival. J Neurosci 19:8945-8953.

Liu A, Niswander LA (2005) Bone morphogenetic protein signalling and vertebrate nervous system development. Nat Rev Neurosci 6:945-954.

Liu X, Hashimoto M, Horii H, Yamaguchi A, Naito K, Yamashita T (2009) Repulsive guidance molecule $\mathrm{b}$ inhibits neurite growth and is increased after spinal cord injury. Biochem Biophys Res Commun 382:795-800.

$\mathrm{Ma} \mathrm{CH}$, Omura T, Cobos EJ, Latrémolière A, Ghasemlou N, Brenner GJ, van Veen E, Barrett L, Sawada T, Gao F, Coppola G, Gertler F, Costigan M, Geschwind D, Woolf CJ (2011) Accelerating axonal growth promotes motor recovery after peripheral nerve injury in mice. J Clin Invest 121:4332-4347.

Ma Q, Sommer L, Cserjesi P, Anderson DJ (1997) Mash1 and neurogenin1 expression patterns define complementary domains of neuroepithelium in the developing CNS and are correlated with regions expressing notch ligands. J Neurosci 17:3644-3652.

Matsunaga E, Nakamura H, Chédotal A (2006) Repulsive guidance molecule plays multiple roles in neuronal differentiation and axon guidance. J Neurosci 26:6082-6088.

Matsuura I, Endo M, Hata K, Kubo T, Yamaguchi A, Saeki N, Yamashita T (2007) BMP inhibits neurite growth by a mechanism dependent on LIM-kinase. Biochem Biophys Res Commun 360:868-873.

Matsuura I, Taniguchi J, Hata K, Saeki N, Yamashita T (2008) BMP inhibition enhances axonal growth and functional recovery after spinal cord injury. J Neurochem 105:1471-1479.

Mills CD, Bitler JL, Woolf CJ (2005) Role of the peripheral benzodiazepine receptor in sensory neuron regeneration. Mol Cell Neurosci 30:228-237.

Mills CD, Allchorne AJ, Griffin RS, Woolf CJ, Costigan M (2007) GDNF selectively promotes regeneration of injury-primed sensory neurons in the lesioned spinal cord. Mol Cell Neurosci 36:185-194.

Moon JI, Birren SJ (2008) Target-dependent inhibition of sympathetic neuron growth via modulation of a BMP signaling pathway. Dev Biol 315:404-417.

Niederkofler V, Salie R, Sigrist M, Arber S (2004) Repulsive guidance molecule (RGM) gene function is required for neural tube closure but not retinal topography in the mouse visual system. J Neurosci 24:808-818.

Niederkofler V, Salie R, Arber S (2005) Hemojuvelin is essential for dietary iron sensing, and its mutation leads to severe iron overload. J Clin Invest 115:2180-2186

Oldekamp J, Krämer N, Alvarez-Bolado G, Skutella T (2004) Expression pattern of the repulsive guidance molecules RGM A-C during mouse development. Gene Expr Patterns 4:283-288.

Oldham MC, Horvath S, Geschwind DH (2006) Conservation and evolution of gene coexpression networks in human and chimpanzee brains. Proc Natl Acad Sci U S A 103:17973-17978.

Oldham MC, Konopka G, Iwamoto K, Langfelder P, Kato T, Horvath S, Geschwind DH (2008) Functional organization of the transcriptome in human brain. Nat Neurosci 11:1271-1282.

Rosen V (2006) BMP and BMP inhibitors in bone. Ann N Y Acad Sci 1068:19-25.

Samad TA, Srinivasan A, Karchewski LA, Jeong SJ, Campagna JA, Ji RR, Fabrizio DA, Zhang Y, Lin HY, Bell E, Woolf CJ (2004) DRAGON: a member of the repulsive guidance molecule-related family of neuronaland muscle-expressed membrane proteins is regulated by DRG11 and has neuronal adhesive properties. J Neurosci 24:2027-2036.

Samad TA, Rebbapragada A, Bell E, Zhang Y, Sidis Y, Jeong SJ, Campagna JA, Perusini S, Fabrizio DA, Schneyer AL, Lin HY, Brivanlou AH, Attisano L, Woolf CJ (2005) DRAGON, a bone morphogenetic protein coreceptor. J Biol Chem 280:14122-14129.

Sánchez-Camacho C, Bovolenta P (2009) Emerging mechanisms in morphogen-mediated axon guidance. Bioessays 31:1013-1025. 
Schwab JM, Monnier PP, Schluesener HJ, Conrad S, Beschorner R, Chen L, Meyermann R, Mueller BK (2005) Central nervous system injuryinduced repulsive guidance molecule expression in the adult human brain. Arch Neurol 62:1561-1568.

Seijffers R, Allchorne AJ, Woolf CJ (2006) The transcription factor ATF-3 promotes neurite outgrowth. Mol Cell Neurosci 32:143-154.

Seijffers R, Mills CD, Woolf CJ (2007) ATF3 increases the intrinsic growth state of DRG neurons to enhance peripheral nerve regeneration. J Neurosci 27:7911-7920.

Severyn CJ, Shinde U, Rotwein P (2009) Molecular biology, genetics and biochemistry of the repulsive guidance molecule family. Biochem J 422:393-403.

Sjöberg J, Kanje M (1990) The initial period of peripheral nerve regeneration and the importance of the local environment for the conditioning lesion effect. Brain Res 529:79-84.

Smith WC (1999) TGF beta inhibitors. New and unexpected requirements in vertebrate development. Trends Genet 15:3-5.

Tsujii M, Akeda K, Iino T, Uchida A (2009) Are BMPs involved in normal nerve and following transection?: a pilot study. Clin Orthop Relat Res 467:3183-3189.

Wu MY, Hill CS (2009) Tgf-beta superfamily signaling in embryonic development and homeostasis. Dev Cell 16:329-343.
Xia Y, Sidis Y, Mukherjee A, Samad TA, Brenner G, Woolf CJ, Lin HY, Schneyer A (2005) Localization and action of Dragon (repulsive guidance molecule b), a novel bone morphogenetic protein coreceptor, throughout the reproductive axis. Endocrinology 146:3614-3621.

Xia Y, Babitt JL, Bouley R, Zhang Y, Da Silva N, Chen S, Zhuang Z, Samad TA, Brenner GJ, Anderson JL, Hong CC, Schneyer AL, Brown D, Lin HY (2010) Dragon enhances BMP signaling and increases transepithelial resistance in kidney epithelial cells. J Am Soc Nephrol 21:666-677.

Xia Y, Cortez-Retamozo V, Niederkofler V, Salie R, Chen S, Samad TA, Hong CC, Arber S, Vyas JM, Weissleder R, Pittet MJ, Lin HY (2011) Dragon (repulsive guidance molecule B) inhibits IL-6 expression in macrophages. J Immunol 186:1369-1376.

Xiao Q, Du Y, Wu W, Yip HK (2010) Bone morphogenetic proteins mediate cellular response and, together with Noggin, regulate astrocyte differentiation after spinal cord injury. Exp Neurol 221:353-366.

Zhao GQ (2003) Consequences of knocking out BMP signaling in the mouse. Genesis 35:43-56.

Zou H, Ho C, Wong K, Tessier-Lavigne M (2009) Axotomy-induced Smad1 activation promotes axonal growth in adult sensory neurons. J Neurosci 29:7116-7123. 